\section{pHYPER, a shRNA vector for high-efficiency RNA interference in embryonic stem cells}

\author{
Soizik Berlivet, Virginie Guiraud, Martin Houlard, and Matthieu Gérard \\ Commissariat à l'Énergie Atomique (CEA), Institut de Biologie et Technologies de \\ Saclay (iBiTecS), Epigenetic Regulation and Cancer Group, Gif-sur-Yvette, France
}

BioTechniques 42:738-743 (June 2007)

doi $10.2144 / 000112454$

RNA interference (RNAi) is a powerful method to generate loss-of-function phenotypes. Plasmid vectors with RNA polymerase III promoters have been developed to express short hairpin RNAs (shRNAs) in mammalian cells. In order to optimize the efficiency of these vectors in embryonic stem (ES) cells, we have constructed and tested several plasmids, based on the H1 promoter, that direct the expression of shRNAs. The original pSUPER vector was used as a reference in this study. This vector drives the expression of shRNAs from a basic 0.2-kb $H 1$ promoter, which exhibits a variable expression when integrated into the genome of ES cells. We used a 2.5-kb mouse genomic fragment containing the $H 1$ promoter to construct a new H1 shRNA vector, $P H Y P E R$. A comparison of this vector with the basic 0.2-kb H1 vector showed that pHYPER directs the synthesis of higher amounts of shRNAs. Using epifluorescence and fluorescent-activated cell sorting (FACS) analysis, we demonstrated that PHYPER is 4-fold more active than the 0.2-kb H1-based vector after integration into the genome of mouse ES cells. We provide a new, improved H1 shRNA vector that is optimized for both transient transfection studies and the generation of stable ES cell lines.

\section{INTRODUCTION}

RNA interference (RNAi) has been used in a wide range of organisms to analyze gene function. RNAi was adapted to mammalian cells following the discovery that transfected small double-stranded RNAs can target the degradation of specific mRNAs (1). Synthetic duplex RNAs have a short half-life and therefore are not adapted for long-term studies or permanent knockdown of gene activity. Plasmid vectors that transcribe short hairpin RNAs (shRNAs) were then developed as alternative tools to suppress gene expression in mammals (2-7). These vectors usually contain the H1 or U6 promoters that transcribe a sequence encoding the sense and antisense RNA strands separated by a short loop. Transcription of this RNA terminates after synthesis of the second strand-at the TTTTT termination signal for RNA polymerase III. The synthesized RNA will fold into a stem-loop precursor, the shRNA, that is cleaved into small inhibitory RNAs (siRNAs) that enter the RNAi cellular machinery (8). The better understanding of the cellular RNAi machinery allowed substantial improvements in the efficiency of shRNA vectors. For instance, the observation of thermodynamic asymmetry in siRNAs $(9,10)$ resulted in the development of rules predicting shRNA sequences with improved efficiencies. Recently, shRNAs that mimic a natural microRNA (miRNA) primary transcript were shown to be more efficient for suppression of gene expression (11). The shRNA technology was successfully applied to a wide series of cell lines, including embryonic stem (ES) cells (12). Understanding the biology of pluripotent ES cells is a great challenge for the future, as these cells might represent the best material for regenerative cell therapy. Loss-offunction analysis of genes using the shRNA method will provide invaluable information on their function in the maintenance of stem cell identity or their capacity to differentiate.

Here, we have set out to optimize the use of shRNA technology in ES cells by testing constructs derived from the $\mathrm{H} 1$ promoter. We show that a new shRNA vector, pHYPER, is 4-fold more active in ES cells than the widely used 0.2-kb H1-based shRNA plasmid. We provide evidence that $\mathrm{pHYPER}$ is highly efficient for the generation of stable knockdown ES cell lines and for short-term transfection experiments.

\section{MATERIALS AND METHODS}

\section{DNA Constructs}

All vectors express a 23-nucleotide hairpin-type shRNA with a 6nucleotide loop. The sequence for the green fluorescent protein (GFP) and p150CAF-1 shRNAs were described in Reference 13 and Reference 14. The construction of pHYPER and pShRNA0.2 plasmids is described in the supplementary materials (available online at www.BioTechniques.com). We used circular plasmids for transient transfection experiments, and linear, XhoI-digested plasmids for the generation of stable knockdown ES cell clones. DNA fragments were agarose gel-purified in order to remove the plasmid sequences (AmpR and Ori) prior to electroporation in ES cells.

\section{Embryonic Stem Cells}

LTP20 cells were derived from blastocysts obtained from $\mathrm{C} 3 \mathrm{H} / \mathrm{HeJ}$ females bred to $129 \mathrm{~Sv}$ males, as previously described (15). LTM7 cells were described previously (14). LTP20 ES cells were transfected by electroporation with a plasmid expressing enhanced GFP (EGFP) under the control of the pCAGGS promoter (16). After a week under hygromycin selection, brightly fluorescent GFP expressing ES colonies were selected for amplification. We further selected ES clones that remained brightly fluorescent during embryoid bodies' formation and differentiation. One of these cell lines, ES-GFP1, was used in all subsequent electroporation experiments.

\section{Epifluorescence Analysis of GFP Depletion by RNA Interference}

Mouse ES cells were cultured as previously described (17). shRNA plasmid vectors were purified using a NucleoSpin ${ }^{\circledR}$ plasmid kit (MachereyNagel, Easton, PA, USA). ES-GFP1 cells $\left(2 \times 10^{7}\right)$ were electroporated $(250$ $\mathrm{V}$ with a capacitance of $500 \mu \mathrm{F}$ ) with 10 
$\mu \mathrm{g}$ shRNA vector, plated, and cultured for $24 \mathrm{~h}$ in the absence of selection. Cells were further cultured for an additional 5 days in the presence of puromycin (2 $\mu \mathrm{g} / \mathrm{mL}$ ). ES cell colonies were observed using a Leica MZFLIII epifluorescence binocular, and pictures were taken using a CoolSNAPTM camera (Princeton Instruments, Trenton, NJ, USA) and its associated software.

\section{Northern Blot Analysis}

ES cells were transfected with pHYPER and pShRNA0.2 and cultured as described in the section entitled Epifluorescence Analysis of GFP Depletion by RNA Interference. RNAs were purified by TRIzol $^{\circledR}$ reagent and chloroform extraction. We used the mirVana ${ }^{\mathrm{TM}}$ miRNA Isolation kit from Ambion (Austin,
TX, USA) to further purify small RNAs. One microgram of the small RNAs' fraction was run onto a $15 \%$ acrylamide/8 $\mathrm{M}$ urea gel and transferred to a Hybond-N+ membrane (GE Healthcare, Piscataway, NJ, USA). Hybridization was performed at $37^{\circ} \mathrm{C}$, in $6 \times$ saline-sodium phosphate-EDTA (SSPE), $0.1 \%$ sodium dodecyl sulfate (SDS), and $2 \times$ Denhart's solution, using a ${ }^{32} \mathrm{P} 5$ '-labeled oligonucleotide complementary to the antisense siRNA of GFP. Hybridization signals were quantified using an Instant Imager (PerkinElmer, Waltham, MA, USA).

\section{Fluorescent-Activated Cell Sorting and Western Blot Analysis}

ES-GFP1 cells were transfected with pHYPER or pShRNA0.2 as described in the section entitled
Northern Blot Analysis. After 5 days of puromycin selection, cells were trypsinized, collected, and washed twice with phosphate-buffered saline (PBS) solution. Untransfected ESGFP1 and LTP20 cells were used as GFP-expressing and nonfluorescent control cells, respectively. Cells were fixed for $10 \mathrm{~min}$ in PBS solution with $4 \%$ paraformaldehyde at room temperature, washed once, and resuspended in PBS solution at $10^{5}$ cells $/ \mathrm{mL}$. The $10^{5}$ cells were analyzed by fluorescent-activated cell sorting (FACS) using a FACSCalibur ${ }^{\mathrm{TM}}$ system (BD Biosciences, San Jose, CA, USA). This experiment was repeated twice and gave identical results. For Western blot analysis, extracts were analyzed using standard procedures. Antibodies against GFP and $\beta$-actin were from Abcam (Cambridge, MA, USA).
A

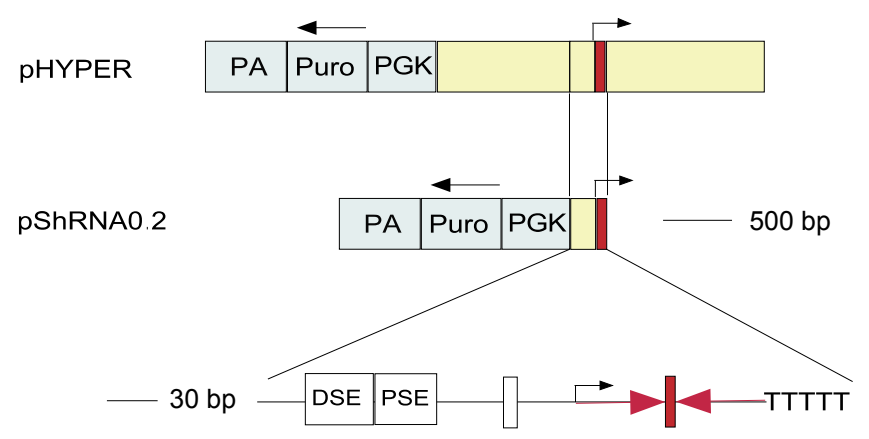

B
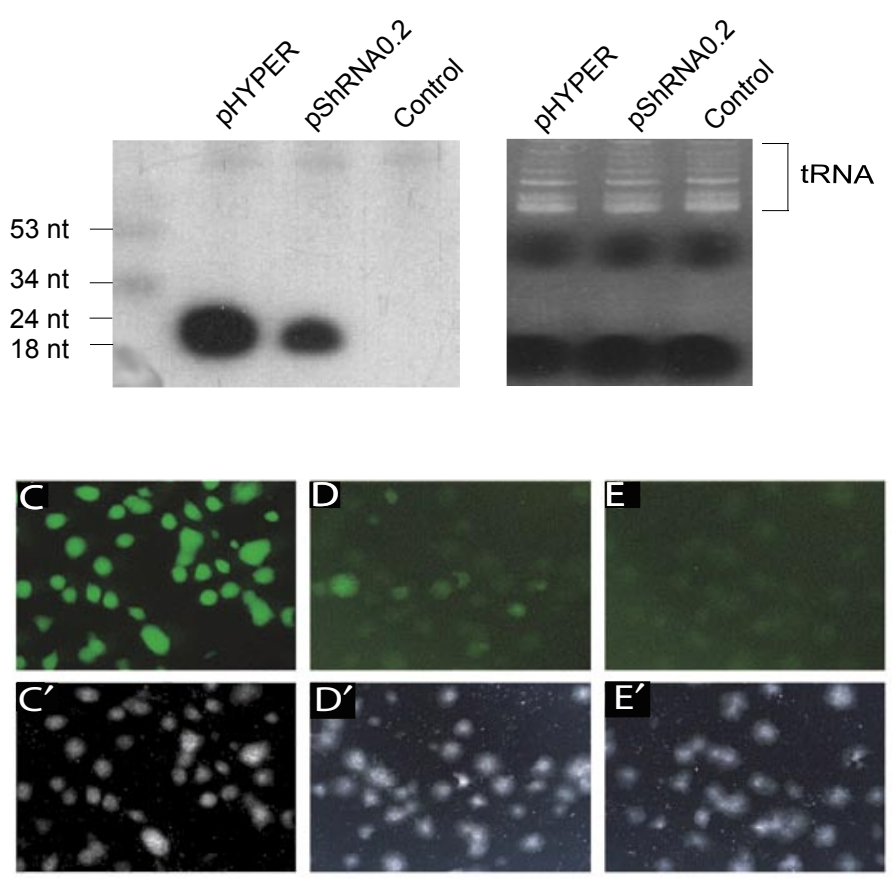

Figure 1. pHYPER triggers potent repression of green fluorescent protein (GFP) expression in embryonic stem (ES) cell colonies. (A) Structure of the vectors used in ES cells' transfection experiments. pHYPER contains $2.5 \mathrm{~kb}$ mouse H1 genomic DNA (yellow) and a puromycin selection cassette (blue) in the opposite orientation with respect of $\mathrm{H} 1$ transcription. In pShRNA0.2, the mouse $\mathrm{H} 1$ promoter is reduced to $0.2 \mathrm{~kb}$ H1 genomic DNA, as in pSUPER (2). This $0.2-\mathrm{kb}$ DNA fragment includes the distal sequence element (DSE), proximal sequence element (PSE), and the TATA box (white square) (19). Both pHYPER and pShRNA0.2 vectors control the transcription of a short hairpin RNA (shRNA) (red) that targets GFP mRNA. PGK, phosphoglycerate-kinase promoter; $\mathrm{pA}$, signal for RNA polymerase II transcription termination. (B) Northern blot analysis of small inhibitory RNA (siRNA) production. ES cells were transfected with pHYPER, pShRNA0.2, and a control pHYPER expressing RNA limited to the sense strand of the shRNA. siRNAs were analyzed using a probe complementary to the siRNA antisense strand (left panel). The positions of an RNA ladder are indicated on the left. The right panel shows RNA stained with ethidium bromide $(\mathrm{EtBr})$ to control the amount of total RNA in each lane. (C-E) Epifluorescence analysis of ES cells transfected with GFP-targeting shRNA plasmid vectors. ES cell colonies were observed under fluorescent $(\mathrm{C}-\mathrm{E})$ or visible light $\left(\mathrm{C}^{\prime}-\mathrm{E}^{\prime}\right)$, at low magnification. (C) Untransfected ES cell colonies are shown as a control. ES cells were transfected with (D) pShRNA0.2 and (E) pHYPER vectors, respectively, and cultured for 5 days in the presence of puromycin. 


\section{Analysis of p150CAF-1 Depletion by RNA Interference}

LTM7 ES cells were transfected by electroporation with pHYPER, seeded onto gelatin-coated microscope slides, and cultured for $24 \mathrm{~h}$ in the absence of selection. Puromycin $(2 \mu \mathrm{g} / \mathrm{mL})$ was added to the medium, and cells were cultured for an additional $48 \mathrm{~h}$. Cells were washed several times with PBS solution and fixed for $20 \mathrm{~min}$ in PBS containing $4 \%$ paraformaldehyde. 4',6-diamidino-2-phenylindole (DAPI) staining and immunofluorescence analysis were performed as described (14).

\section{RESULTS AND DISCUSSION}

In mouse, large transgenes containing extensive flanking genomic sequences around a gene of interest often give more faithful expression patterns than small transgenes (18). These effects are linked to the presence of additional regulatory elements or insulators that can be located kilobases away from the promoter region. We tested whether this general rule, which is clearly relevant for RNA polymerase II promoters, might also apply for the H1 RNA polymerase III-driven promoter. We designed a new $\mathrm{H} 1$ vector, pHYPER, which contains 2500 bp mouse genomic DNA flanking the H1 transcription start site $(1250 \mathrm{bp}$ $5^{\prime}$ and 1250 bp $3^{\prime}$ of H1 start site; see Figure 1A). pHYPER was deleted of the H1 RNA coding sequence, which was replaced by a shRNA that targets the degradation of GFP-mRNA. We choose a shRNA-GFP sequence that was previously validated for efficient GFP-mRNA degradation (13). We tested this construct in comparison with a 210-bp mouse $\mathrm{H} 1$ promoter vector, pShRNA0.2, whose H1 promoter is similar in composition and length to the human $\mathrm{H} 1$ promoter used in the pSUPER vector (2). Both human and mouse 210-bp H1 DNA fragments comprise the three cisacting elements that were shown to be essential for full $\mathrm{H} 1$ promoter activity in COS-7 cells: $(i)$ the TATA box, (ii) the proximal sequence element (PSE), and (iii) the distal sequence element (DSE) containing an octamer motif and a Staf binding site (19). The pHYPER and pShRNA0.2 vectors were electroporated into an ES cell line expressing a GFP reporter gene (see the Materials and Methods section). ES clones were allowed to grow under appropriate puromycin selection. After 6 days in culture, puromycin-resistant ES cell colonies had stably integrated the shRNA expressing vectors into their genome (data not shown). We performed a Northern blot analysis to quantify the expression of siRNAs specific for GFP in these cells. This experiment revealed a 2-fold increase in the amount of 20 nucleotides siRNA species in cells transfected with pHYPER, compared with pShRNA0.2 (Figure 1B). Identical results were obtained with RNAs prepared after a shorter culture period (3 days; data not shown). These siRNAs are expected to be produced by the cleavage and processing of shRNAs expressed from the $\mathrm{H} 1$ promoters. A Southern blot analysis verified that the number of copies of $\mathrm{pHYPER}$ and pShRNA0.2 vectors inserted into the genome of ES cells was not significantly different (data not shown). We next performed an epifluorescence analysis of transfected ES cells, which revealed that the two vectors gave easily discernable patterns. The large pHYPER construct gave a potent repression of GFP expression, with $>85 \%$ of ES colonies displaying a complete absence of fluorescence (Figure 1D). In contrast, most ES cell colonies transfected with the small pShRNA0.2 showed residual GFP expression (Figure 1C). We then used FACS analysis to quantify these observations. ES cells were transfected with pHYPER and pShRNA0.2 vectors directed against GFP, as previously described. After 5 days of culture under puromycin selection, the whole population of ES cell colonies from

D

E
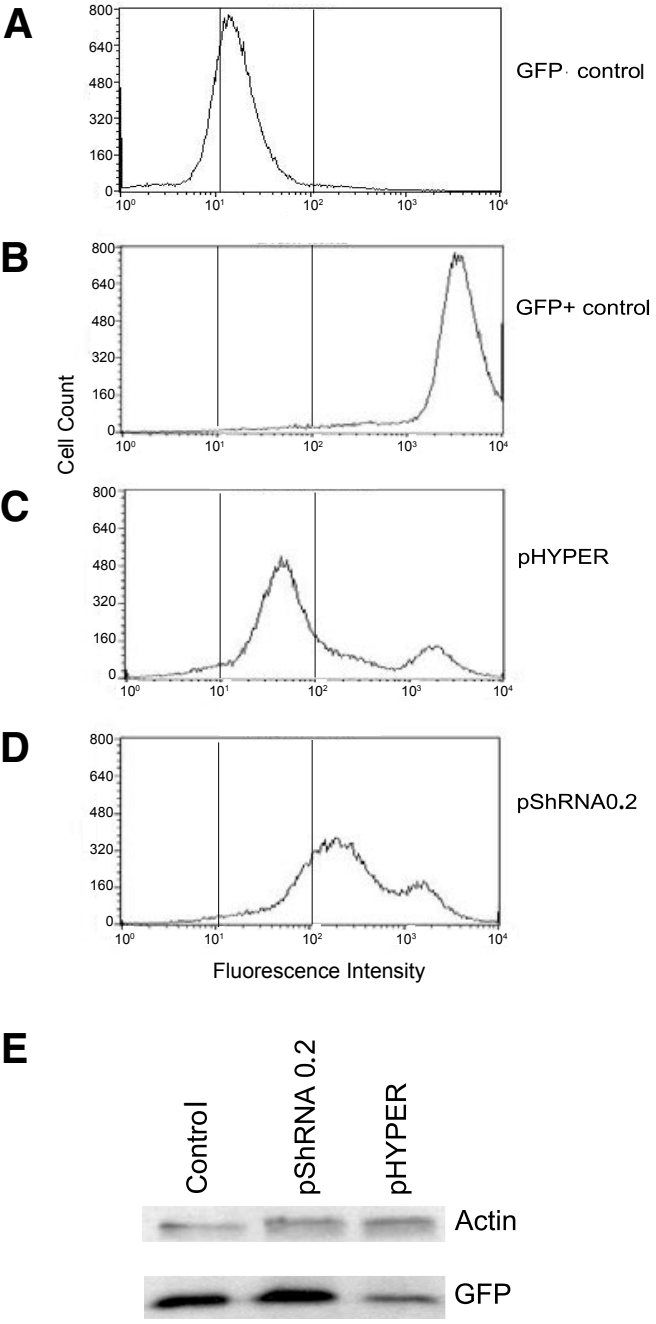

Figure 2. Fluorescent-activated cell sorting (FACS) and Western blot analyses of green fluorescent protein (GFP) knockdown using pShRNA0.2 and pHYPER plasmid vecors. Nonfluorescent and GFP-expressing embryonic stem ES) cells are shown as controls in panel A and panel B, respectively. Transfection of GFP-expressing ES cells with (C) pHYPER and (D) pShRNA0.2 results in reduced fluorescence evels; y-axis, cell count (arbitrary units). (E) Western blot nalysis. $\mathrm{x}$-axis, fluorescence intensity.

a 10-cm culture dish (about 3000 independent colonies per dish) was homogenized by trypsin, and 100,000 cells were analyzed by FACS (Figure 2, $\mathrm{C}$ and D). Based on GFP fluorescence intensity, we could distinguish three categories of cells for both vectors, classified as high-, medium-, or lowefficiency GFP knockdown. Of the cells transfected with pHYPER, 67\% were in the high-efficiency knockdown category, in contrast to $<23 \%$ for pShRNA0.2 (Figure 2, C and D). Conversely, $18 \%$ pHYPER-transfected, and $60 \%$ pShRNA0.2-transfected cells were in the medium-efficiency 


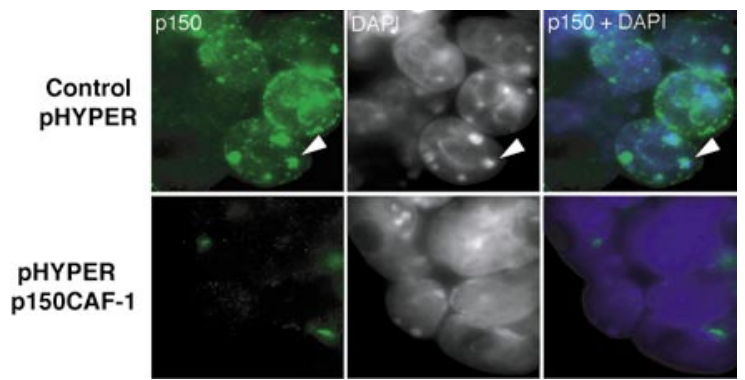

Figure 3. Knockdown of an essential gene using PHYPER in a transient transfection protocol. Upper panels: (Left): immunodetection of p150CAF-1 (green) in embryonic stem (ES) cells expressing a control short hairpin RNA (shRNA) that targets green fluorescent protein (GFP) (control pHYPER). (Middle): DNA staining with the fluorochrome 4',6-diamidino-2-phenylindole (DAPI) shows the normal appearance of heterochromatin domains, revealed as bright foci (arrowhead). (Right): The merge between p150CAF-1 fluorescence and DAPI-stained DNA (in blue) reveals the presence of p150CAF-1 at the level of heterochromatin domains during DNA replication. Lower panels: (Left): Immunodetection of p150CAF-1 (green) in ES cells transfected with a p150CAF-1 targeting pHYPER vector reveals an efficient knockdown. DAPI staining (middle) reveals the disruption of heterochromatin foci following p150CAF-1 depletion. (Right): Merging of p150CAF-1 immunodetection and DAPI signals.

knockdown category. Finally, a similar proportion $(14 \%$ and $16 \%$, respectively) of pHYPER- and pShRNA0.2-transfected cells were in the low-efficiency GFP repression category (Figure 2). In comparison with control GFP-expressing cells (Figure 2B), the majority of transfected ES cells had an average fluorescence intensity reduction of 60 -fold with pHYPER, and only 15-fold with pShRNA0.2. A Western blot analysis confirmed that the results of the FACS analysis were well correlated with the levels of GFP protein in the cells (Figure 2E). Thus, pHYPER is about 4-fold more potent than pShRNA0.2. This increased efficiency of pHYPER, which transcribes a higher level of shRNA than the small $\mathrm{H} 1$ vector, might not only be due to the nature of the sequence of the large $\mathrm{H} 1$ promoter, but could also benefit from an increased distance between $\mathrm{H} 1$ and phosphoglycerate-kinase (PGK) promoter (which drives the expression of the puromycin resistance gene; see Figure 1A).

We next tested the capacity of pHYPER to down-regulate gene expression in transient transfection experiments. In this assay, we choose to target the mRNA encoding the large subunit of chromatin assembly factor-1 (p150CAF-1). This factor is required for mouse ES cell survival, and its genetic ablation results in an easily discernable phenotype (14). ES cells were transfected by electroporation with a pHYPER vector that targets p150CAF-1 mRNA and cultured for 24 $h$ in the absence of selection. Cells were further cultured for an additional $48 \mathrm{~h}$ period in the presence of puromycin ( $2 \mu \mathrm{g} / \mathrm{mL})$, and ES cell colonies were analyzed by immunofluorescence (IF) with an anti-p150CAF-1 antibody. This experiment revealed robust $\mathrm{p} 150 \mathrm{CAF}-$ 1 depletion in $>85 \%$ of the puromycinselected ES cells (Figure 3). Loss of p150CAF-1 function in these cells was confirmed by the disruption of heterochromatin domains, as detected by DAPI staining (Figure 3), a phenotype that we initially discovered in embryos homozygous for a null p150CAF-1 allele (14). A minority of cells $(<15 \%)$ do not repress p150CAF-1 expression efficiently, suggesting that the shRNA vector is inactivated in these cells despite the puromycin selection. An additional 24-h culture period under puromycin selection resulted in the death of cells expressing the p150CAF1 shRNA, but not a control shRNA that targets GFP, revealing that p150CAF-1 is essential for long-term cell viability (data not shown). Thus, the pHYPER vector can be used in transient experiments to study the function of essential genes in ES cells.

In conclusion, the pHYPER vector is dedicated to both the generation of stable ES cell lines and short-term transfection studies in ES cells. Several promoters were previously compared in transient transfection assays for their capacity to silence a reporter gene with shRNA-mir (11). Silva et al. showed that the U6 shRNA and cytomegalovirus (CMV) promoters provided a 2-fold more consistent repression than the human $\mathrm{H} 1$ promoter (11). Here, we demonstrate that the $2.5-\mathrm{kb} \mathrm{H} 1$ promoter of $\mathrm{pHYPER}$ confers a 4-fold enhanced repression when compared with the $0.2-\mathrm{kb} \mathrm{H} 1$ promoter. These data identify pHYPER as the most potent shRNA expressing vector identified to date. In conclusion, pHYPER will improve the panel of available RNAi tools to study gene function in ES cells and other mammalian cell lines.

\section{ACKNOWLEDGMENTS}

We thank S. Jounier and $H$. Humbertclaude for the preparation of feeder cells; U. Rogner, C. Mann, F. Boussouar, and E. Heard for reading the manuscript; J. Acker for advice on RNA analysis; J.C. Amé for the gift of mouse Hl genomic sequences; L. Larue for the pCAGGS plasmid; G. Almouzni for the p150 antibody; and P. Carbon for discussion. This work was supported by the Commissariat à l'Énergie Atomique and the Association pour la Recherche sur le Cancer.

\section{COMPETING INTERESTS STATEMENT}

The authors declare no competing interests.

\section{REFERENCES}

1. Elbashir, S.M., J. Harborth, W. Lendeckel, A. Yalcin, K. Weber, and T. Tuschl. 2001. Duplexes of 21-nucleotide RNAs mediate RNA interference in cultured mammalian cells. Nature 411:494-498.

2. Brummelkamp, T.R., R. Bernards, and R. Agami. 2002. A system for stable expression of short interfering RNAs in mammalian cells. Science 296:550-553.

3.Paddison, P.J., A.A. Caudy, E. Bernstein, G.J. Hannon, and D.S. Conklin. 2002. Short hairpin RNAs (shRNAs) induce sequencespecific silencing in mammalian cells. Genes Dev. 16:948-958.

4.Paul, C.P., P.D. Good, I. Winer, and D.R. Engelke. 2002. Effective expression of small interfering RNA in human cells. Nat. Biotechnol. 20:505-508.

5.Sui, G., C. Soohoo, B. Affar el, F. Gay, Y. Shi, and W.C. Forrester. 2002. A DNA vector-based RNAi technology to suppress gene expression in mammalian cells. Proc Natl Acad Sci USA. 99:5515-5520. 
6. Miyagishi, M. and K. Taira. 2002. U6 promoter-driven siRNAs with four uridine $3^{\prime}$ overhangs efficiently suppress targeted gene expression in mammalian cells. Nat. Biotechnol. 20:497-500.

7. Lee, N.S., T. Dohjima, G. Bauer, H. Li, M.J. Li, A. Ehsani, P. Salvaterra, and J. Rossi. 2002. Expression of small interfering RNAs targeted against HIV-1 rev transcripts in human cells. Nat. Biotechnol. 20:500-505.

8. Valencia-Sanchez, M.A., J. Liu, G.J. Hannon, and R. Parker. 2006. Control of translation and mRNA degradation by miRNAs and siRNAs. Genes Dev. 20:515-524.

9. Schwarz, D.S., G. Hutvagner, T. Du, Z Xu, N. Aronin, and P.D. Zamore. 2003. Asymmetry in the assembly of the RNAi enzyme complex. Cell 115:199-208.

10. Khvorova, A., A. Reynolds, and S.D. Jayasena. 2003. Functional siRNAs and miRNAs exhibit strand bias. Cell 115:209216.

11.Silva, J.M., M.Z. Li, K. Chang, W. Ge, M.C. Golding, R.J. Rickles, D. Siolas, G. Hu, et al. 2005. Second-generation shRNA libraries covering the mouse and human genomes. Nat. Genet. 37:1281-1288.

12. Kunath, T., G. Gish, H. Lickert, N. Jones, T. Pawson, and J. Rossant. 2003. Transgenic RNA interference in ES cell-derived embryos recapitulates a genetic null phenotype. Nat. Biotechnol. 21:559-561.

13. Fritsch, L., L.A. Martinez, R. Sekhri, I. Naguibneva, M. Gerard, M. Vandromme, L. Schaeffer, and A. Harel-Bellan. 2004. Conditional gene knock-down by CRE-dependent short interfering RNAs. EMBO Rep. 5:178-182.

14. Houlard, M., S. Berlivet, A.V. Probst, J.P. Quivy, P. Hery, G. Almouzni, and M. Gerard. 2006. CAF-1 is essential for heterochromatin organization in pluripotent embryonic cells. PLoS Genet 2:e181.

15. Abbondanzo, S.J., I. Gadi, and C.L. Stewart. 1993. Derivation of embryonic stem cell lines. Methods Enzymol. 225:803-823.

16. Niwa, H., K. Yamamura, and J. Miyazaki. 1991. Efficient selection for high-expression transfectants with a novel eukaryotic vector. Gene 108:193-199.

17. Tessarollo, L. 2001. Manipulating mouse embryonic stem cells. Methods Mol. Biol. 158:47-63.

18. Gerard, M., D. Duboule, and J. Zakany. 1993. Structure and activity of regulatory elements involved in the activation of the Hoxd11 gene during late gastrulation. EMBO J. 12:3539-3550.

19. Myslinski, E., J.C. Ame, A. Krol, and P. Carbon. 2001. An unusually compact external promoter for RNA polymerase III transcription of the human H1RNA gene. Nucleic Acids Res. 29:2502-2509.
Received 3 October 2006; accepted 1 March 2007.

Address correspondence to Matthieu Gérard, SBIGeM/LREGE, Bat 142, CEA Saclay, 91191 Gif-sur-Yvette Cedex, France. e-mail: matthieu.gerard@cea.fr

To purchase reprints of this article, contact: Reprints@BioTechniques.com 